\title{
Does the robot have a role in Radical Cystectomy?
}

Pramit Khetrapal1,2, John D. Kelly1,2, James W.F. Catto ${ }^{2,3}$, Nikhil Vasdev 4

1. Division of Surgery \& Interventional Science, University College London, London

2. Surgical \& Interventional Trials Unit (SITU), Division of Surgery \& Interventional Science, University College London, London, NW1 2FD, UK

3. Academic Urology Unit, University of Sheffield, Sheffield, S10 2RX, UK

4. Hertfordshire and Bedfordshire Urological Cancer Centre at the Lister Hospital, Stevenage, Hertfordshire, UK / *School of Life and Medical Sciences, University of Hertfordshire, Hatfield, UK

Corresponding Author:

Pramit Khetrapal

Division of Surgery \& Interventional Science,

University College London,

43-45 Foley Street,

London NW1 2FD, UK

Tel: +44(0)2076796490

Fax: +44(0)20 76796470

Email:p.khetrapal@ucl.ac.uk

Word count: 935 
Between 2014 and 2015, 3,742 radical cystectomies (RC) were performed in the UK. The majority of these were open operations, and only $25 \%$ were performed with robot assistance[1]. These data contrast starkly with the picture in Radical Prostatectomy (RP), for which most operations are robot assisted (79.4\% of the 7,673 in 2016). Given that most pelvic surgeons have access to robotic facilities (as shown by the RP trends) and urologists are typically early adopters, one must question why many surgeons have yet to be convinced by RARC. This question is particularly perplexing given that RC is a more morbid operation than RP and most patients with bladder cancer are considerably less fit than the average man with prostate cancer, and therefore reductions in morbidity are especially rewarding in this cohort.

The ongoing debate amongst cystectomists has merit on both sides. ORC is less expensive, whereas RARC offers reduced blood loss and a potentially quicker recovery. Small randomised studies have shown similar complication rates and peri-operative morbidity[2], and questioned the oncologic efficacy of RARC. The health economics of robotic surgery is another key concern, as benefits from RARC are needed to offset the significantly higher operating costs. Mixed reports regarding costanalysis have been published, but no randomised data are available[3].

This debate has been re-ignited by the recent publication of the RAZOR trial [4]. RAZOR is a multicentre non-inferiority randomised controlled trial (RCT) comparing RARC to ORC. In the trial 350 patients across 15 medical centres in the USA were recruited, including 150 and 152 patients who underwent RARC and ORC respectively. The authors reported a 2-year progression free survival of $72 \cdot 3 \%(95 \% \mathrm{Cl} 64.3$ to 78.8$)$ and $71 \cdot 6 \%(95 \% \mathrm{Cl} 63.6$ to 78.2$)$ in the RARC and ORC groups respectively (difference $0.7 \%, 95 \% \mathrm{Cl}-9.6 \%$ to $10.9 \% ; \mathrm{p}=0.001$ ). This is an important statement as oncological equivalence is necessary to justify using the robotic platform. However, oncologic equivalence may be insufficient in causing large scale adoption of the robotic platform for RC. 
In line with contemporary reports [2], RAZOR reported that RARC had significantly reduced blood loss and transfusion rates, but longer operating times. RAZOR did not provide a cost analysis for RARC vs ORC due to varying costs of RC across centres, but they reported a small albeit significant difference in length of stay between RARC and ORC ( 6 and 7 days, $p=0.0216)$. There was no difference in complication rates. A question arising from these results is whether RAZOR reflects the full potential benefit of RARC and it is noted that the urinary diversion for all cases in robotic arm was performed extracorporeally (eRARC), which means that there was a conversion to open surgery for each case. Could conversion to open surgery negate many of the potential benefits of minimal access surgery? In contrast, intracorporeal RARC (iRARC) whereby both the extirpative and the diversion are performed robotically, is completely minimal access. As acknowledged by authors of the RAZOR trial[4], iRARC may improve peri-operative recovery when compared to eRARC.

Furthermore, there are no accepted definitions of surgical experience for RC. RAZOR required surgeons to have performed 10 RCs in the year prior to trial recruitment. In contrast, less than $5 \%$ of RCs in the UK are performed by surgeons undertaking $<8$ RCs/year, and the majority of RCs $(56.5 \%)$ are performed by high-volume surgeons undertaking $\geq 30$ RCs/year. While RAZOR's requirement of $10 \mathrm{RCs}$ in the year prior is a minimum requirement and it is likely that most surgeons did significantly more than that, setting the bar this low - regardless of technique and outcomes - could potentially have allowed novice surgeons to operate on trial patients. Median RARC operating times in RAZOR were $>7$ hours, and the Pasadena consensus recommends that experienced surgeons should aim to complete the procedure between 5-6 hours[5]. According to the consensus definition, surgeons are on their learning curve for the first 30 cases - and it is possible that a subset of robotic surgeons in RAZOR were still on their learning curve. Indeed, the median time for eRARC is similar to retrospective data reported by Hussein et al.[6], which collected data from all surgeons without any 
criteria on experience, albeit with the possibility of selection bias, and reported a median operating time of 400 minutes across 1, 031 cases. However, it must be noted that there is also no standard definition for 'operating time'. In the RAZOR trial, "room in to room out" time was used, whereas the Pasadena consensus statement did not specify if they used the same definition, or console time, anaesthetic time, surgical time (difference between closure and knife-to-skin time).

With the results of RAZOR, there is still no discernible benefit for RARC over ORC. Oncological equivalence is a reassuring finding, but does not provide a rationale for the comparatively expensive RARC. These findings reaffirm the NHS England's clinical commissioning policy on robotic cystectomy, that NHS England will not routinely commission robotic assisted surgery for bladder cancer. It is likely that any benefit of RARC will be in the peri-operative recovery, and this needs to be compared in an RCT, with high volume surgeons in both arms, enhanced recovery and intracorporeal diversion.

To this end, a phase III multicentre randomised controlled trial to compare the efficacy of Robotically Assisted Radical Cystectomy (RARC) and intracorporeal urinary diversion with Open Radical Cystectomy (ORC) in patients with bladder cancer (the iROC trial) is currently recruiting in high volume centres across the UK[7]. Primary outcome of the iROC trial is to assess difference in days alive and out of hospital for patients undergoing RARC and ORC. The iROC will further help address key questions on the role of both RARC and intracorporeal urinary robotic diversion in current clinical practice from the perspective of a health economic analysis.

While RAZOR represents an important milestone for robotic surgeons, more evidence is required to understand if RARC should be adopted as the new definitive standard treatment for bladder cancer. 
Oncological equivalence is an important aspect to justify a rationale for RARC, but is not enough to sway policy decisions in favour of the relatively expensive procedure. We hope that results of the iROC trial will help inform the urological community of any difference in peri-operative outcomes between truly minimal access RARC and ORC. 


\section{Reference list}

1. Khadhouri S, Miller C, Cresswell J, et al. The BAUS radical cystectomy audit 2014/2015 - an update on current practice and an analysis of the effect of centre and surgeon case volume. Eur Urol Suppl [Internet]. Elsevier B.V.; 2017;16(3):e473-4.

2. Tan WS, Khetrapal P, Tan WP, Rodney S, Chau M, Kelly JD. Robotic Assisted Radical Cystectomy with Extracorporeal Urinary Diversion Does Not Show a Benefit over Open Radical Cystectomy: A Systematic Review and Meta-Analysis of Randomised Controlled Trials. Schwentner C, editor. PLoS One [Internet]. 2016 Nov 7;11(11):e0166221. Available from: http://dx.plos.org/10.1371/journal.pone.0166221

3. Mmeje CO, Martin AD, Nunez-Nateras R, Parker AS, Thiel DD, Castle EP. Cost analysis of open radical cystectomy versus robot-assisted radical cystectomy. Curr Urol Rep [Internet]. 2013 Feb 25 [cited 2018 Apr 19];14(1):26-31. Available from: http://link.springer.com/10.1007/s11934-012-0292-7

4. Parekh DJ, Reis IM, Castle EP, et al. Robot-assisted radical cystectomy versus open radical cystectomy in patients with bladder cancer (RAZOR ): an open-label, randomised, phase 3, non-inferiority trial. Lancet [Internet]. Elsevier Ltd; 2018;391(10139):2525-36. Available from: http://dx.doi.org/10.1016/S0140-6736(18)30996-6

5. Wilson TG, Guru K, Rosen RC, et al. Best practices in robot-assisted radical cystectomy and urinary reconstruction: Recommendations of the pasadena consensus panel. Eur Urol [Internet]. European Association of Urology; 2015;67(3):363-75. Available from: http://dx.doi.org/10.1016/j.eururo.2014.12.009

6. Hussein AA, May PR, Jing Z, et al. Outcomes of Intracorporeal Urinary Diversion after RobotAssisted Radical Cystectomy: Results from the International Robotic Cystectomy Consortium. J Urol. 2018;199(5):1302-11.

7. Catto JW., Khetrapal P, Ambler G, et al. Robot Assisted Radical Cystectomy with intracorporeal urinary diversion versus Open Radical Cystectomy (iROC): protocol for a randomised controlled trial with internal feasibility study. BMJ Open. 2018. 